\title{
ChemComm
}

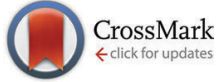

Cite this: Chem. Commun., 2014, 50, 15228

Received 14th September 2014, Accepted 17th October 2014

DOI: $10.1039 / \mathrm{c} 4 \mathrm{cc} 07271 \mathrm{e}$

www.rsc.org/chemcomm

\section{Nuclear spin circular dichroism in fullerenes: a computational study}

\author{
Michal Straka, ${ }^{* a}$ Petr Štěpánek, ${ }^{a}$ Sonia Coriani ${ }^{b}$ and Juha Vaara*c
}

\begin{abstract}
In the recently proposed phenomenon, nuclear spin-induced circular dichroism (NSCD), collective magnetisation of nuclei induces circular dichroism in a light beam passing through a molecular sample. Here we present the first computational predictions of NSCD for fullerenes $\mathrm{C}_{60}$ and $\mathrm{C}_{70}$. We show that the NSCD signal is nucleus-specific, like in NMR spectroscopy. Thus, NSCD may provide a new and promising, high-resolution observable for experimental identification of chemical compounds.
\end{abstract}

Nuclear magneto-optic spectroscopy (NMOS) techniques couple optical measurements of birefringence and dichroism with the use of the magnetisation of atomic nuclei to cause these changes in the polarisation state of light. NMOS methods have been a subject of growing interest and rapid development, due to their potential to provide a nucleus-specific spectroscopic signal. ${ }^{1}$

An experimentally successful example of NMOS is nuclear spin optical rotation (NSOR), in which controlled magnetisation of nuclear spins along the light beam induces optical rotation of linearly polarised light (LPL) passing through the sample..$^{a, d, e, j, k}$ In the pilot study of Savukov, Lee, and Romalis ${ }^{1 a}$ the NSOR signal was observed for the first time in liquid Xe and water samples. This work inspired further experimental ${ }^{1 d, e, j, k}$ and theoretical ${ }^{1 c, g, h, j}$ studies. It was also shown theoretically that different nuclear sites within a single molecule should provide different NSOR signals in analogy to nuclear magnetic resonance (NMR). ${ }^{1 c j, j, n}$

A promising, novel candidate for spectroscopic characterisation of molecules is the nuclear spin circular dichroism (NSCD) recently formulated and implemented by Vaara et al. ${ }^{1 q}$ The principle of NSCD is illustrated in Fig. 1. In this technique, the magnetically polarised nuclei induce ellipticity in linearly polarised light passing through a molecular sample, in a manner similar

\footnotetext{
${ }^{a}$ Institute of Organic Chemistry and Biochemistry, Academy of Sciences of the Czech Republic, Flemingovo nám. 2., CZ-16610, Praha 6, Czech Republic. E-mail: straka@uochb.cas.cz

${ }^{b}$ Dipartimento di Scienze Chimiche e Farmaceutiche, Università degli Studi di Trieste, Via L. Giorgieri 1, I-34127 Trieste, Italy

${ }^{c}$ NMR Research Group, Department of Physics and Chemistry, P.O. Box 3000, FIN-90014 University of Oulu, Finland. E-mail: juha.vaara@iki.fi
}

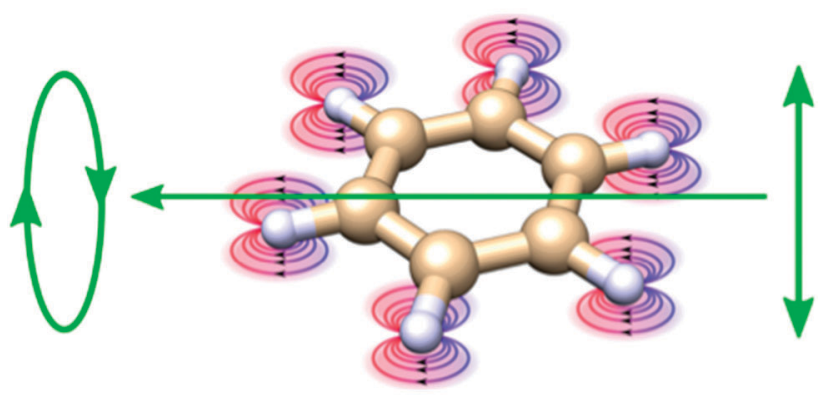

Fig. 1 Schematic illustration of NSCD, where ellipticity (ellipse on the left) is induced in the linearly polarised light (arrow on the right) by collective magnetisation of atomic nuclei (represented by local magnetic fields on $\mathrm{H}$ atoms).

to magnetic circular dichroism (MCD), ${ }^{2}$ where the $\mathrm{CD}$ effect is induced by an external magnetic field oriented along the light path. In practice, the MCD spectrum is obtained by measuring the differential absorption of the left- and right-circularly polarised components of LPL.

NSCD and MCD are "mathematically" similar in their 3rd-order response theory expressions, where the dynamic molecular polarizability is modified by a static magnetic interaction. ${ }^{1 q}$ However, the two phenomena are physically different. In MCD, the static magnetic interaction is the global Zeeman interaction with the external magnetic field, whereas in NSCD the local hyperfine interaction is involved. This provides NSCD with the exciting possibility of gaining local, nucleus-specific information. The signal obtained in MCD originates from the entire molecule. On the other hand, due to the hyperfine interactions, NSCD has potential to offer distinction of chemical environments, with atom-site specific signals, similar to NMR but by using optical detection.

In the proposed ${ }^{1 q}$ but not yet performed NSCD experiment, nuclei are first magnetised (e.g., by using NMR magnets and coils) and then the differential absorption of the light passing through the sample is measured. To obtain the chemical resolution by the NSCD technique, two principal means suggested for $\operatorname{NMOS}^{1 c}$ can be used: (i) wavelength tuning to localised excitations 
and (ii) selective magnetisation using NMR techniques. It has been suggested that by tuning the laser wavelength in NSOR experiments to the specific resonances, the NSOR signal can be localised to the different chromophores in molecules. ${ }^{1 c, q}$ Numerous approaches for inducing and controlling the magnetisation of nuclei are known in NMR spectroscopy and have already been discussed and successfully tested in NSOR experiments. ${ }^{1 a, d, e, j, k}$

While the construction of the first experimental NSCD apparatus is on its way, it is crucial to provide a basic understanding of the NSCD observable to guide the future experiments. Here we present the first computational investigation $\dagger$ of NSCD for fullerenes $\mathrm{C}_{60}$ and $\mathrm{C}_{70}$, to illustrate the potential of the method. The calculated ${ }^{13} \mathrm{C}$ NSCD spectra are analysed and compared to the available MCD spectra, recorded and calculated previously. ${ }^{3}$

The predicted $\dagger{ }^{13} \mathrm{C}$ NSCD spectrum for $\mathrm{C}_{60}$ is compared to the MCD one ${ }^{3 a}$ in Fig. 2. Since all carbons in $\mathrm{C}_{60}$ are equivalent, the total ${ }^{13} \mathrm{C}$ NSCD signal in Fig. 2 was obtained by multiplying the calculated signal of a single carbon nucleus by the factor of 60 .

The signs and relative intensities of the ${ }^{13} \mathrm{C}$ NSCD bands do not fully correlate with those of the related MCD bands; some of the features have the same sign for both NSCD and MCD (Fig. 2 near 210 and $245 \mathrm{~nm}$ ), while some have opposite signs (Fig. 2 near $320 \mathrm{~nm}$ ). The positive NSCD band near $250 \mathrm{~nm}$ shows a rather strong relative intensity as compared to MCD one. This leads to a different overall shape of the NSCD and MCD signals.

Let us now turn to $\mathrm{C}_{70}$ fullerene with five distinct carbon atom types (Fig. 3). They have the following degeneracies: $10 \times \mathrm{C}_{\mathrm{a}}, 10 \times \mathrm{C}_{\mathrm{b}}, 20 \times \mathrm{C}_{\mathrm{c}}, 20 \times \mathrm{C}_{\mathrm{d}}$, and $10 \times \mathrm{C}_{\mathrm{e}}$. This degeneracy is taken into account in Fig. 4 and 5, where the corresponding, calculated ${ }^{13} \mathrm{C}$ NSCD intensities for single nuclei have been multiplied by the factors of $10,10,20,20$, and 10 for $\mathrm{C}_{\mathrm{a}}-\mathrm{C}_{\mathrm{e}}$, respectively.

The MCD signal for $\mathrm{C}_{70}$ does not match the NSCD data in shape, neither for the individual $\mathrm{C}_{\mathrm{a}}-\mathrm{C}_{\mathrm{e}}$ signals, nor for the total

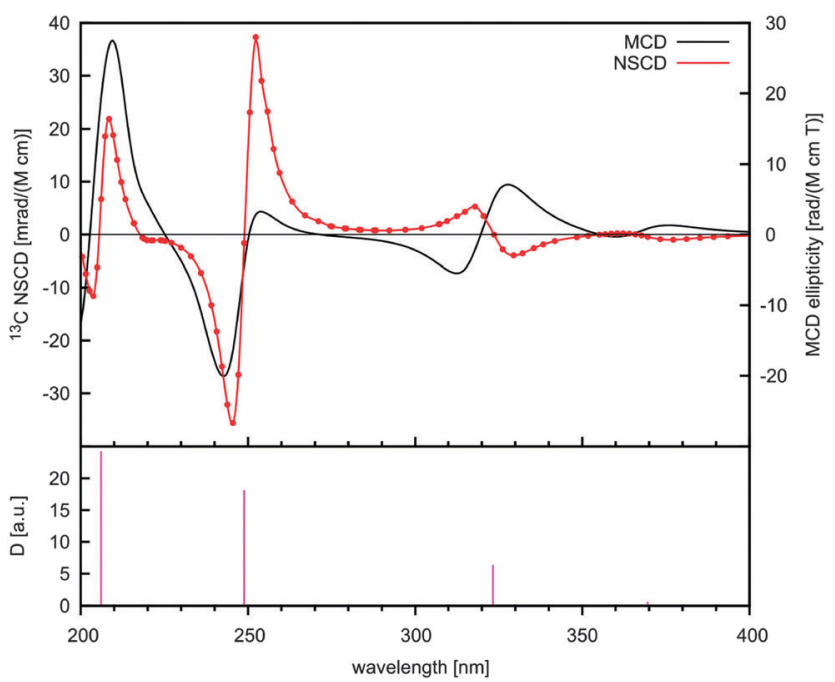

Fig. 2 The calculated NSCD (B3LYP/def2-SV+P) $\dagger$ and MCD (B3LYP/6-31G*) $)^{3 a}$ signals for $C_{60}$. The excitation wavelengths and transition dipole strengths $D$ $(B 3 L Y P / d e f 2-S V+P) \dagger$ are shown in the lower panel for clarity.

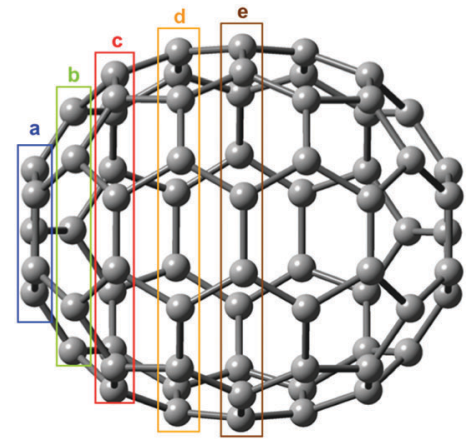

Fig. 3 The five distinct symmetry-equivalent carbon types $C_{a}-C_{e}$ in $C_{70}$.

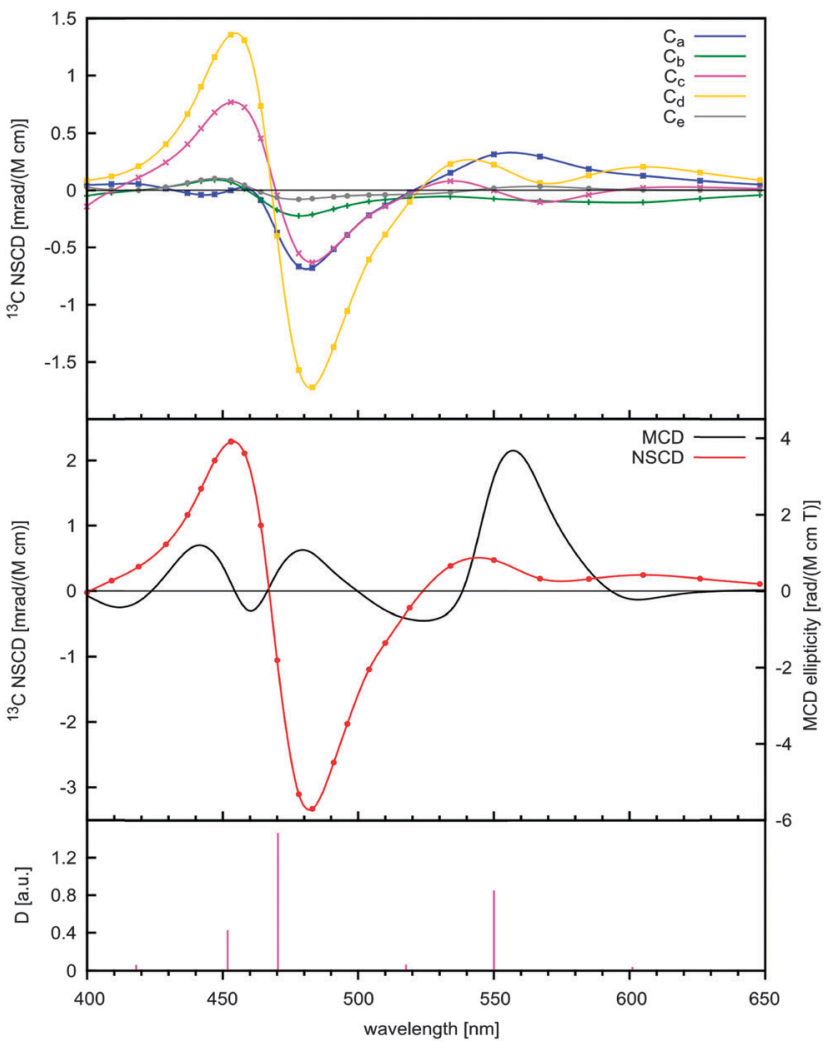

Fig. 4 The calculated ${ }^{13} \mathrm{C}$ NSCD (B3LYP/def2-SV+P, upper panel) $\dagger$ for the $\mathrm{C}_{\mathrm{a}}-\mathrm{C}_{\mathrm{e}}$ nuclear sites and the MCD (B3LYP/6-31G*, middle panel) ${ }^{3 a}$ in $\mathrm{C}_{70}$. The total ${ }^{13} \mathrm{C}$ NSCD signal (middle panel) is obtained as a sum of the signals for $\mathrm{C}_{\mathrm{a}}-\mathrm{C}_{\mathrm{e}}$. The excitation wavelengths and transition dipole strengths $D(B 3 L Y P / d e f 2-S V+P) \dagger$ are shown in the bottom panel. The spectral region $400-630 \mathrm{~nm}$ is shown.

NSCD signal, Fig. 4 and 5. The individual $C_{a}-C_{e}$ signals provide clearly distinct spectral patterns.

The intensity of the NSCD signals is rather different for the distinct carbon types at certain wavelengths, regardless of the degree of degeneracy. This is nicely seen in the NSCD signals for $\mathrm{C}_{\mathrm{a}}$ and $\mathrm{C}_{\mathrm{e}}$ in Fig. 4, both arising from 10-fold degenerate carbon types. Near 480 and $550 \mathrm{~nm}, \mathrm{C}_{\mathrm{e}}$ gives a rather weak signal as compared to $\mathrm{C}_{\mathrm{a}}$, while the opposite occurs near $270 \mathrm{~nm}$. There, the signal corresponding to $\mathrm{C}_{\mathrm{e}}$ is rather strong and $\mathrm{C}_{\mathrm{a}}$ amounts only to about a third of it. More examples can be found in Fig. 5, e.g., near 270 and $370 \mathrm{~nm}$. 


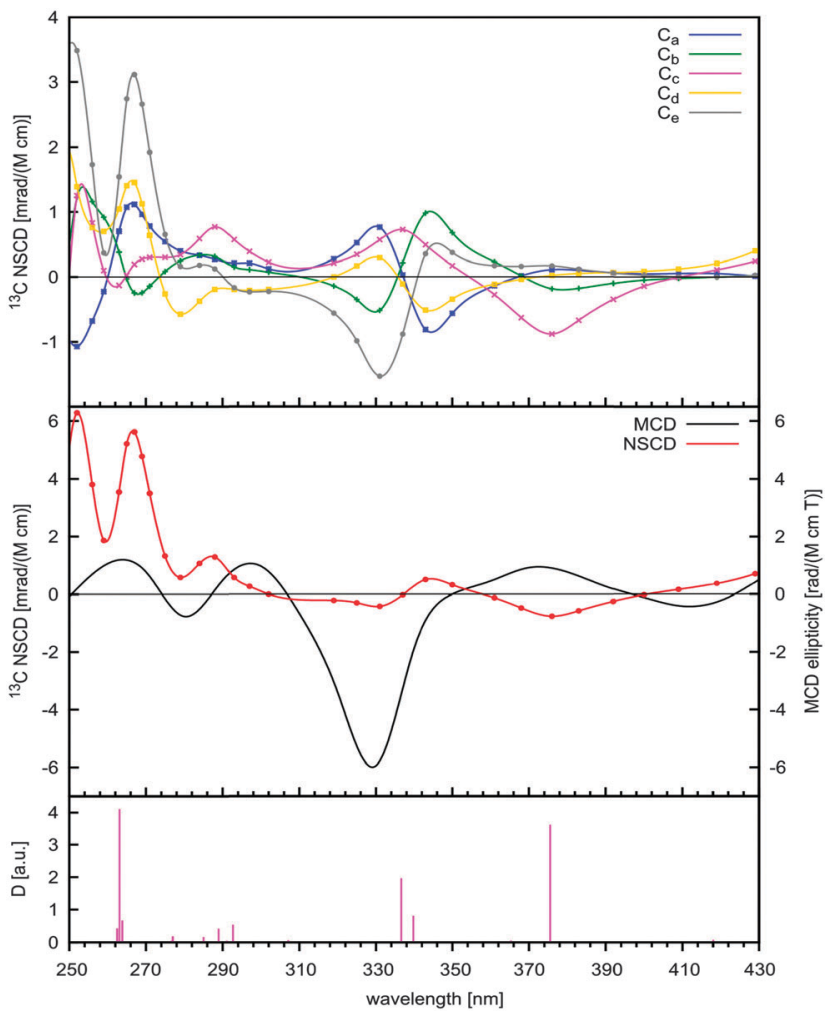

Fig. 5 The calculated ${ }^{13} \mathrm{C}$ NSCD (B3LYP/def2-SV+P, upper panel) $\uparrow$ for the $\mathrm{C}_{a}-\mathrm{C}_{\mathrm{e}}$ nuclear sites and the MCD (B3LYP/6-31G*, middle panel) ${ }^{3 a}$ in $\mathrm{C}_{70}$. The total ${ }^{13} \mathrm{C}$ NSCD signal (middle panel) is obtained as a sum of the signals for $C_{a}-C_{e}$. The excitation wavelengths and transition dipole strengths $D$ $(\mathrm{B} 3 \mathrm{LYP} / \mathrm{def} 2-\mathrm{SV}+\mathrm{P}) \dagger$ are shown in the bottom panel. The spectral region $250-430 \mathrm{~nm}$ is shown.

Perhaps the most interesting observation in Fig. 4 and 5 is that the positions of the peaks and couplets on the wavelength axis are actually shifted off the corresponding resonances and this shift also depends on the carbon type, even when the corresponding ${ }^{13} \mathrm{C}$ NSCD bands have the same sign and similar intensity. For example, in the region of 320-380 nm (Fig. 5) the negative couplet for $\mathrm{C}_{c}$ is found at a much higher wavelength than those for $\mathrm{C}_{\mathrm{a}}$ and $\mathrm{C}_{\mathrm{d}}$. This is due to the different strengths of the NSCD signal at the three main resonances of 338, 340 and $376 \mathrm{~nm}$ (bottom panel of Fig. 5), which causes the different shapes and positions of these couplets. At some resonances that are hardly visible in the UV/vis spectra due to a small transition dipole strength, a large MCD signal may nevertheless emerge. ${ }^{3}$ This is also observed in NSCD, for example near 290 and $250 \mathrm{~nm}$. This feature is also one of the reasons for the differential positions of the bands for the different carbon types. For example, the strong absorption calculated at $550 \mathrm{~nm}$ (exp. $544 \mathrm{~nm})^{3 a}$ is accompanied by a weak one near $518 \mathrm{~nm}$, hardly visible in the experimental UV/vis spectra. Due to the linewidth and intensity effects, the maxima and minima for different carbon types are actually seen at different frequencies, compare, e.g., carbons $\mathrm{C}_{\mathrm{a}}$ and $\mathrm{C}_{\mathrm{d}}$ near $550 \mathrm{~nm}$ in Fig. 4 .

The total ${ }^{13} \mathrm{C}$ NSCD signal for $\mathrm{C}_{70}$ shown in Fig. 4 and 5 is also rather different from the MCD signal and constitutes a candidate for the first possible experimental NSCD measurements, before the resolution of individual carbon signals is reached.

In summary, signals appropriate to the recently proposed nuclear spin-induced circular dichroism (NSCD) experiment were calculated for $\mathrm{C}_{60}$ and $\mathrm{C}_{70}$ fullerenes. We have shown that the NSCD signal is nucleus-specific, i.e., it may vary for distinct carbon sites. The signals from the distinct carbon atom types differ by their shape, band/couplet position on the wavelength axis, and intensity. Signals that are weak in absorption spectra can become visible in NSCD. The site-specificity suggests that NSCD spectroscopy may be amenable for experimental highresolution identification of chemical compounds. In a recent study, closely related NSOR results were reported to provide nucleusspecific information on finite-size flakes of graphenic materials, including their defects ( $\mathrm{sp}^{2}$ islands in an $\mathrm{sp}^{3}$ environment and vice versa). ${ }^{1 r}$ Similar applications can be foreseen for NSCD.

The project was supported by the Research Council of University of Oulu (MS and JV), the Czech Science Foundation (14-03564S, MS), the Czech Academy of Sciences (RVO-61388963, MS and PS), and the Academy of Finland (JV). The COST-CMTS Action CM1002 "Convergent Distributed Environment for Computational Spectroscopy (CODECS)" is acknowledged. Computational resources were provided by CSC-IT center for Science (Espoo, Finland) and the Finnish Grid Initiative as well as by CERIT Scientific Cloud, R\&D Program, no. CZ.1.05/ 3.2.00/08.0144.

\section{Notes and references}

$\dagger$ Methods. For a detailed account of the theoretical foundations and implementation of NSCD we refer to ref. $1 q$. Calculations on $\mathrm{C}_{60}$ and $\mathrm{C}_{70}$ fullerenes were carried out for molecules in their equilibrium groundstate geometries in vacuo, thus neglecting all vibronic and solvent effects. The structures were optimised using the Turbomole code, version 6.3.1., ref. 4 , the BP86 density functional ${ }^{5}$ and the def-TZVP 6 carbon basis set. NSCD calculations were done using the complex polarisation propagator (CPP) approach implemented ${ }^{1 q}$ in the 2014 release version of the Dalton program package, ${ }^{7}$ which exploits the CPP response equation solver of ref. 8. The B3LYP density functional ${ }^{9}$ was employed in the calculations. The selection of B3LYP was strongly motivated by its good performance, using methodology similar to that of present work, for both the absorption and MCD spectra of $\mathrm{C}_{60}$ and $\mathrm{C}_{70}$, ref. 3a. B3LYP performed well also for the calculations of ${ }^{13} \mathrm{C}$ NMR shielding in fullerenes. ${ }^{10}$ The basis set for NSCD calculations was constructed by augmenting the def2-SVP basis set ${ }^{11}$ by an sp set of diffuse functions $\left(\alpha_{s}=0.04363394\right.$ and $\left.\alpha_{p}=0.05089538\right)$. This combination gives a good balance of accuracy and computational cost, and is referred to as def2-SV+P. The empirical linewidth parameter required by the CPP approach was set to $1000 \mathrm{~cm}^{-1}$ (0.00456 a.u.), following previous experience, e.g., ref. $1 q$ and 3 . The step between the consecutive frequencies was initially set equal to 0.0025 a.u. and then refined whenever needed.

1 (a) I. M. Savukov, S. K. Lee and M. V. Romalis, Nature, 2006, 442, 1021-1024; (b) T.-t. Lu, M. He, D.-m. Chen, T.-j. He and F.-c. Liu, Chem. Phys. Lett., 2009, 479, 14-19; (c) S. Ikäläinen, M. V. Romalis, P. Lantto and J. Vaara, Phys. Rev. Lett., 2010, 105, 153001; (d) D. Pagliero, W. Dong, D. Sakellariou and C. A. Meriles, J. Chem. Phys., 2010, 133, 154505; (e) D. Pagliero and C. A. Meriles, Proc. Natl. Acad. Sci. U. S. A., 2011, 108, 19510-19515; $(f)$ G.-h. Yao, M. He, D.-m. Chen, T.-j. He and F.-c. Liu, Chem. Phys., 2011, 387, 39-47; $(g)$ T. S. Pennanen, S. Ikäläinen, P. Lantto and J. Vaara, J. Chem. Phys., 2012, 136, 184502; (h) S. Ikäläinen, P. Lantto and J. Vaara, J. Chem. Theory Comput., 2012, 8, 91-98; (i) G.-h. Yao, M. He, D.-m. Chen, T.-j. He and F.-c. Liu, ChemPhysChem, 2012, 13, 1325-1331; $(j)$ J. Shi, S. Ikäläinen, J. Vaara 
and M. V. Romalis, J. Phys. Chem. Lett., 2013, 4, 437-441; (k) I. M. Savukov, H. Y. Chen, T. Karaulanov and C. Hilty, J. Magn. Reson., 2013, 232, 31-38; (l) L.-j. Fu, A. Rizzo and J. Vaara, J. Chem. Phys., 2013, 139, 181102; (m) L.-j. Fu and J. Vaara, J. Chem. Phys., 2013, 138, 204110; (n) F. Chen, G.-h. Yao, T.-j. He, D.-m. Chen and F.-c. Liu, Chem. Phys., 2014, 435, 57-61; (o) L.-j. Fu and J. Vaara, ChemPhysChem, 2014, 15, 2337-2350; (p) L.-j. Fu and J. Vaara, J. Chem. Phys., 2014, 140, 024103; $(q)$ J. Vaara, A. Rizzo, J. Kauczor, P. Norman and S. Coriani, J. Chem. Phys., 2014, 140, 134103; (r) J. Vähäkangas, P. Lantto and J. Vaara, J. Phys. Chem. C, 2014, 118, 23996-24005.

2 R. W. Mason, A practical guide to magnetic circular dichroism spectroscopy, John Wiley \& Sons, Inc., New Jersey, 2007.

3 (a) P. Stěpánek, M. Straka, V. Andrushchenko and P. Bouř, J. Chem. Phys., 2013, 138, 151103; (b) T. Fahleson, J. Kauczor, P. Norman and S. Coriani, Mol. Phys., 2013, 111, 1401-1404.

4 R. Ahlrichs, M. Bär, M. Häser, H. Horn and C. Kölmel, Chem. Phys. Lett., 1989, 162, 165-169.

5 (a) A. D. Becke, Phys. Rev. A: At., Mol., Opt. Phys., 1988, 38, 3098-3100; (b) J. P. Perdew, Phys. Rev. B: Condens. Matter Mater. Phys., 1986, 33, 8822.
6 C. Schäfer, C. Huber and R. Ahlrichs, J. Chem. Phys., 1994, 100, 5829.

7 (a) K. Aidas, C. Angeli, K. L. Bak, V. Bakken, R. Bast, L. Boman, O. Christiansen, R. Cimiraglia, S. Coriani and P. Dahle, et al., Wiley Interdiscip. Rev.: Comput. Mol. Sci., 2014, 4, 269-284; (b) Dalton, a molecular electronic structure program, Release DALTON2014 (2014), see http://daltonprogram.org.

8 (a) J. Kauczor, P. Jørgensen and P. Norman, J. Chem. Theory Comput., 2011, 7, 1610-1630; (b) J. Kauczor and P. Norman, J. Chem. Theory Comput., 2014, 10, 2449-2455.

9 (a) A. D. Becke, J. Chem. Phys., 1993, 98, 5648-5652; (b) C. Lee, W. Yang and R. G. Parr, Phys. Rev. B: Condens. Matter Mater. Phys., 1988, 37, 785; (c) P. J. Stephens, F. J. Devlin, C. F. Chabalowski and M. J. Frisch, J. Phys. Chem., 1994, 98, 11623-11627.

10 (a) J. Kaminský, M. Buděšínský, S. Taubert, P. Bouř and M. Straka, Phys. Chem. Chem. Phys., 2013, 15, 9223; (b) T. Kupka, M. Stachów, E. Chełmecka, K. Pasterny, M. Stobińska, L. Stobiński and J. Kaminský, J. Chem. Theory Comput., 2013, 9, 4275-4286.

11 F. Weigend and R. Ahlrichs, Phys. Chem. Chem. Phys., 2005, 7, 3297-3305. 